\title{
Talocalcaneal coalition combined with flatfoot in children: diagnosis and treatment: a review
}

\author{
Binghua Zhou', Kanglai Tang ${ }^{1 *}$ and Mark Hardy ${ }^{2^{*}}$
}

\begin{abstract}
Talocalcaneal coalition often leads to a flatfoot deformity in children. Previous reports have uncovered many aspects of tarsal coalition and flatfoot respectively, including the etiology, clinical presentation, and diagnostic imaging, as well as treatment. However, the optimum surgical procedure for talocalcaneal coalition combined with flatfoot has not been definitively determined. The nonconformity of treatment options is due to our incomplete knowledge of biomechanics, diagnosis, and indication of treatment for talocalcaneal coalition with flatfoot. The objectives of this review are to provide an overview of the current knowledge about etiology, biomechanics, classification, diagnosis, and treatment options for talocalcaneal coalitions with flatfoot and highlight its therapies in children.
\end{abstract}

Keywords: Coalition, Flatfoot, Children, Diagnosis, Treatment

\section{Introduction}

Tarsal coalition is an abnormal connection between the tarsal bones, which is thought to result from a congenital failure of differentiation in the developing fetal foot. The incidence of tarsal coalition has been reported from less than $1 \%$ to as high as $13 \%$ [1-3]. Patients frequently have more than one coalition in the same foot and $50 \%$ of patients are bilateral. The most common coalitions involve the talocalcaneal and calcaneonavicular bone [1]. In some series, talocalcaneal coalitions account for up to $48 \%$ of all tarsal coalition cases [4]. This does not appear to be a sex predilection of tarsal coalitions [5].

The calcaneus is held in a valgus position as ossification progresses with a talocalcaneal coalition, which leads to a rigid flatfoot deformity. Subtalar motion is markedly restricted in the talocalcaneal coalitions but is moderately limited in calcaneonavicular and talonavicular coalitions. Bony coalition can be detected on routine radiographs. However, fibrous or cartilaginous coalitions may be difficult to visualize with standard radiograph. Computed tomography (CT) and magnetic resonance (MR) imaging are invaluable for assessment of tarsal coalitions.

\footnotetext{
* Correspondence: kanglaitang1968@gmail.com; hardy.mark@icloud.com Equal contributors

${ }^{1}$ Department of Orthopedic Surgery, Third Military Medical University Affiliated Southwest Hospital, Gaotanyan Str. 30, Chongqing 400038, People s Republic of China

${ }^{2}$ Department of Foot \& Ankle Surgery, HealthSpan Physicians Group, 10 Severance Circle, Cleveland Heights, OH 44118, USA
}

Resection of coalition is generally accepted for an intractably painful talocalcaneal tarsal coalition. Despite the possibility that symptoms may be relieved with coalition resection, normal foot alignment and muscular imbalances cannot be restored with resection alone [6]. The treatment for talocalcaneal coalitions with flatfoot in children continues to be a topic of debate. In this review, we present the existing knowledge on the etiology, classification, diagnosis, and treatment of the talocalcaneal coalitions with flatfoot and debate whether there is an optimal surgical procedure.

\section{Clinical symptoms}

Talocalcaneal coalitions with flatfoot generally start as a painless decrease in the range of motion of a joint and will often progress to a symptomatic rigid deformity [1]. Patients with talocalcaneal coalition and flexible flatfoot deformity are often asymptomatic with a rectus foot type. Talocalcaneal coalition becomes symptomatic during childhood as the coalition ossifies and restricts subtalar motion [7]. Pain presents in the sinus tarsi when the middle facet of talocalcaneal joint has a solid osseous bridge. Once the posterior facet of talocalcaneal joint demonstrates degenerative changes; the pain is often located medially, inferior to the medial malleolus, at the site of coalition [8]. Talocalcaneal coalitions with a rigid flatfoot deformity will often show symptoms between 1216 years of age $[9,10]$. However, some children between the ages of 
610 years were found to have completely ossified talocalcaneal coalitions [9].

Talocalcaneal coalition is the most common cause of peroneal spastic flatfoot. Peroneal spastic flatfoot is a syndrome typically characterized by limited tarsal joint motion, a clonus response of the evertors, and a pes planus deformity [11]. Muscle spasm in severe patients may also involve the extensor digitorum longus and peroneus tertius muscles; adaptive shortening of peroneal tendons can lead to reflex spasms [12]. Clinical symptoms of the tarsal coalition frequently follow a sequence of sprains or other minor injuries [1,2]. Because the absence of inflammatory cells has been reported in histopathological studies, it is believed that pain is secondary to mechanical stress arising from the periosteum surrounding the ossifying coalition [13]. Collectively, peroneal spasm is the most common clinical symptom in patients with talocalcaneal coalition and associated flatfoot. Most patients have a consistent pain located at the sinus tarsi and over the sustentaculum tali. However, the location of pain varies and pain can be diffuse throughout the hindfoot complex.

\section{Etiology}

Proposed theories related to the etiology of tarsal coalitions can be described as being either congenital or acquired [1]. The most widely accepted of congenital coalitions is the LeBouqs theory that autosomal dominant inheritance led to the failure of differentiation of embryonic mesenchymal tissue [1,14]. A previous report showed that Asian people have a higher rate of tarsal coalitions. The pattern of foot coalitions in Native American samples can be traced back to the early appearance and widespread geographic distribution populations migrating from Asia [15]. Recently, Pro250Arg point mutation in fibroblast growth factor receptor 3 gene was thought to result in a tarsal coalition [16]. A much higher percentage of coalitions than anticipated in either the general population or the injured athletic population reinforced that there may be an underlying anatomic predisposition [3]. Talocalcaneal coalitions also can be acquired by degenerative joint disease, inflammatory arthritis, infection, fibular hemimelia, and clubfoot deformities [1].

\section{Biomechanics}

During a normal gait cycle, the subtalar joint experiences rotatory and gliding motion of the talus against the calcaneus. Following heel strike, the talocalcaneal joint assumes relative external rotation and valgus with maximum calcaneal eversion during the first half of the stance. During this stance segment, the calcaneus also dorsiflexes and the Chopart articulation abducts. Then, gradual internal rotation and varus takes place during the remaining stance and swing phase [17]. The subtalar joint functions as a torque convertor, which transmits forces from the tibia to the foot. Mobility of the transverse tarsal joint is determined by the position of the subtalar joint, when the hindfoot is everted into a valgus position, and the transverse tarsal joint is unlocked. Talocalcaneal coalitions restrict mobility of the subtalar joint severely, which lead to increased frontal plane range of motion due to compensatory adaptation or lateral ligamentous laxity [18]. In addition, with the presence of calcaneal valgus, the fibula may come into contact with the lateral calcaneal wall during weight bearing and develop a pseudoarticulation [19].

The importance of achieving normal subtalar kinematics during stance cannot be overemphasized. Normal subtalar kinematics attenuates ground reaction impact forces and limits subtalar eversion, which increases the magnitude of the impact loading experienced during locomotion. Tarsal coalition feet displayed reduced peak pressure and loading at the region of the fifth metatarsal head [6]. Electromyography revealed consistent abnormal activity in the peroneal, gastrocnemius, and soleus muscles on both the operated foot and the contralateral side, including either prolonged monophasic activity or biphasic activity [6].

Many of the operative procedures described for tarsal coalitions may improve gait but they do not fully restore a normal physiologic gait. Therefore, foot function assessment preoperatively and postoperatively is critical to determine the efficacy of various surgical procedures for patients with tarsal coalition. Feet with talocalcaneal coalition had significantly higher medial midfoot pressures during walking and running preoperatively, compared to the asymptomatic extremity. However, medial midfoot pressures showed no significant difference during walking but significantly higher during running after the resection of coalition [20]. The feet continued to be subjected to increased loading and torque in their subtalar and adjacent articulations after resection of coalition [17]. In conclusion, isolated resection of a tarsal coalition fails to restore normal subtalar joint kinematics.

\section{Classification}

Classification systems have been proposed for coalitions based on their anatomic location. For talocalcaneal coalition, most coalitions occur in the middle facet; however, posterior and anterior facet coalitions have also been reported [21]. Coalitions can be further classified into fibrous, cartilaginous, and osseous as to the type of tissue making up the coalitions [22]. Bourdet identified four patterns of flatfoot [23]. These classifications are primarily descriptive for coalition and flatfoot respectively, which afford little information for coalition with flatfeet. Downey introduced and added three important 
parameters into the articular classification system for tarsal coalition, including: the patient age, articular involvement, and the extent of the secondary arthritic changes [24].

Type IA: extra-articular coalition without secondary arthritis;

Type IB: extra-articular coalition with secondary arthritis;

Type IIA: extra-articular coalition without secondary arthritis;

Type IIB: extra-articular coalition with secondary arthritis.

This classification system combined the characters of both coalition and flatfoot well. It can be used as a framework to discuss recommended surgical procedures and their clinical results.

\section{Diagnosis}

Congenital talocalcaneal coalition is often overlooked in children who first present with foot and ankle pain [8]. Additionally, the differential diagnosis of talocalcaneal coalition is easy to misinterpret in skeletal samplings [25]. Therefore, careful physical examination and radiograph evaluation are required to correctly diagnose the condition.

\section{Physical examination}

A thorough physical examination determines if the causes of the symptoms are related solely to the coalition, secondary arthritic changes, or flatfoot deformity. Tarsal coalitions with a rigid flatfoot are characterized by peroneal muscle spasms and a painful, rigid valgus deformity [12]. The physical examination should focus on these symptoms.

On clinical examination, patients generally have mild to deep pain within the subtalar joint with limitation of passive range of motion of this joint. During open kinetic chain examination, these patients have a valgus position of the hindfoot, as well as an equinus position at the level of the ankle, forefoot pronation, and loss of medial longitudinal arch height [8]. If the coalition is unilateral, this position is easily observed relative to the contralateral limb.

A toe-raise test can be used to assess the mobility of the hindfoot. The patient is asked to rise on the toes of both feet simultaneously. In the rigid coalition foot, the heel remains everted or vertical because of the rigid nature of the condition; conversely, in a flexible foot, the heel will invert and reduce because the equinus component is negated, allowing the extrinsic musculature to realign the foot [14]. When supination of the weightbearing foot generates only a limited external rotation of the tibia, tarsal coalition must be assumed [26]. The step-forward Hubscher maneuver was introduced as an effective means of evaluating the flexibility and reducibility of a pes planovalgus deformity by negating the effects of a gastrocnemius or gastrocnemius-soleus equinus. In stance, the hallux is manually dorsiflexed and the leg is externally rotated, which will demonstrate a restored arch in the flexible/reducible foot, via windlass loading of the plantar fascia. Conversely, this maneuver will fail to restore the arch in a rigid foot [8].

\section{Radiographic imaging}

Bony coalition can be detected on routine radiographs. Talocalcaneal coalitions had an $88 \%$ diagnostic specificity radiographically [27]. The $\mathrm{C}$ sign has been described as an important radiographic sign of talocalcaneal coalition which is a C-shaped line created by the outline of the talar dome and the inferior margin of the sustentaculum tali [26]. A middle facet coalition in the subtalar joint results in uneven medial forces through the middle facet, rather than the posterior facet, which normally bears the load of these forces. Therefore, it is seen that there is

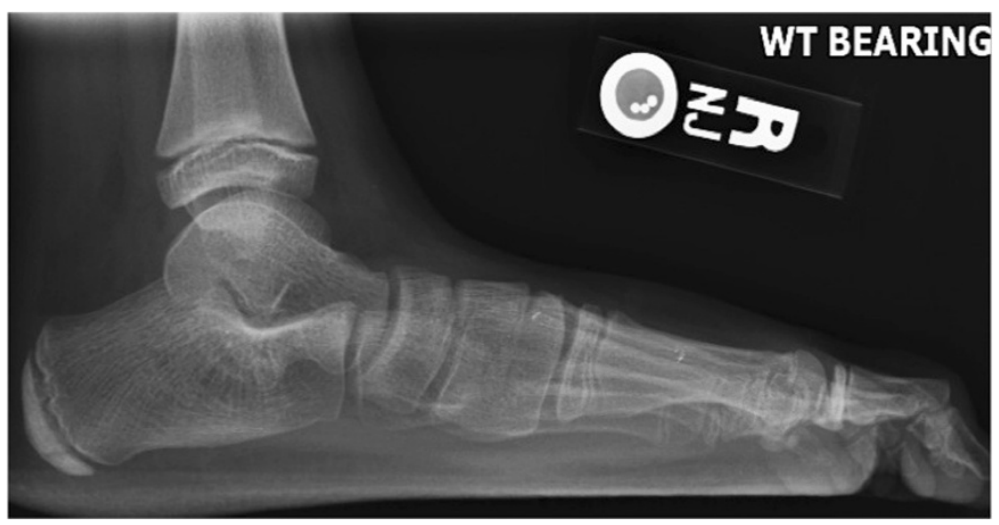

Figure 1 The lateral X-ray image shows the C sign. 


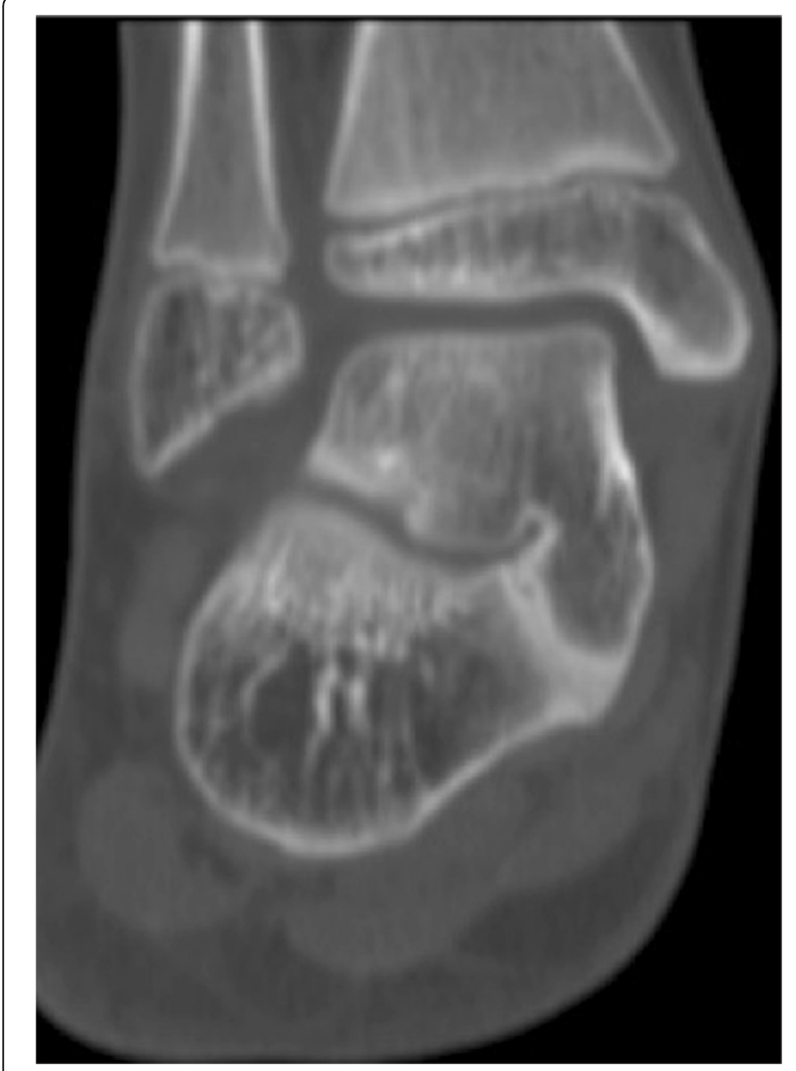

Figure 2 The image of CT shows a bony talocalcaneal coalition.

irregularity of the middle facet and hypertrophy of the sustentaculum in the lateral radiograph. The anatomicpathologic results showed that the $\mathrm{C}$ sign is a bony bridge between the talar dome and sustentaculum tali, in combination with a prominent inferior border of the sustentaculum tali (Figure 1). Brown et al. have shown that the $\mathrm{C}$ sign may be more indicative of collapsing flatfoot deformity than coalition [26]. In addition, the fibula may come into contact with the lateral calcaneal wall during weight bearing and develop a pseudoarticulation in the presence of calcaneal valgus [19].

Fibrous or cartilaginous talocalcaneal coalitions may be difficult to visualize on the three standard radiographic views of the foot. CT and MR imaging will help to delineate the diagnosis and guide the surgical treatment plan $[9,28]$. CT and MR imaging allow differentiation of osseous from nonosseous coalitions and depict the extent of joint involvement as well as secondary degenerative changes [27] (Figure 2). On MR images, bone marrow edema, abnormal articular orientation, and joint space narrowing were frequently identified adjacent to the abnormal joint. However, Wilde showed that there was a significant association between the outcome scores and the area of coalition [29]. Talocalcaneal coalitions with an associated flatfoot deformity may be detected with advanced imaging when the physical exam and standard radiographs are equivocal.

\section{Treatment options}

The goal of conservative treatment for patients with talocalcaneal coalition and flatfoot is to limit subtalar and midtarsal joint range of motion in an effort to reduce pain and muscle spasms. Conservative treatments include arch supports, a short-leg walking cast, immobilization in neutral or a slight varus position, and anti-inflammatory medications $[2,7]$. Thirty percent of the patients were pain free after 6 weeks of cast treatment in one study [30]. Failure of conservative treatment in symptomatic talocalcaneal coalitions with flatfoot usually mandates a surgical intervention.

Currently, surgical techniques including bar resection with or without interposition in the resection gap, subtalar joint arthrodesis, and also extra-articular arthrodesis in the sinus tarsi have been reported [31,32]. Though, Khoshbin reported that no association was noted between the size of talocalcaneal coalition with respect to outcome scores [33]. It is generally accepted to perform
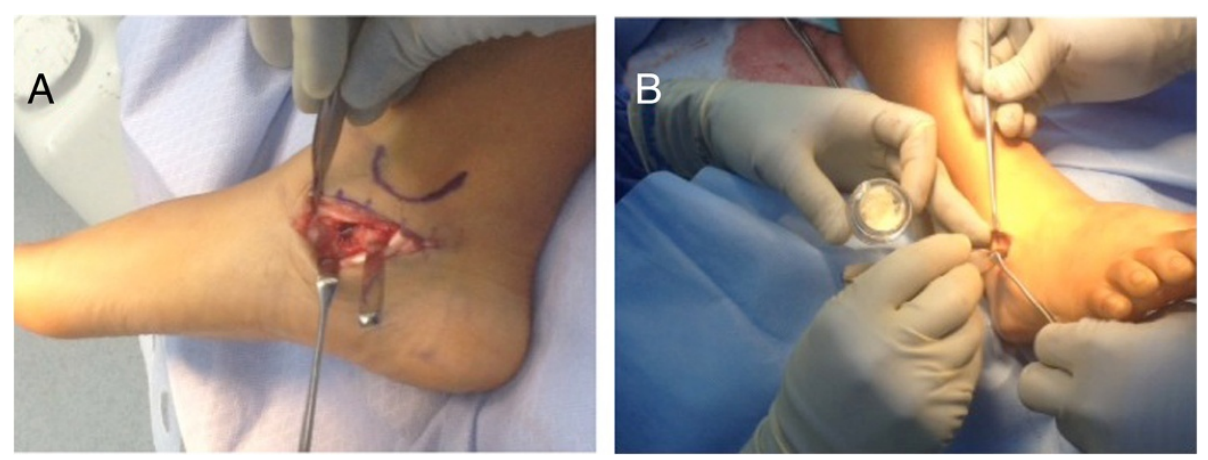

Figure 3 Incision for the exposure of the subtalar joint and the coalition. (A) shows the medial incision for the resection of coalition; (B) shows the lateral incision for sinus tarsi arthrodesis with Cloward spinal graft. 
a single resection of coalition for intractably painful small talocalcaneal coalition that is associated with a wide, healthy posterior facet and minimal valgus deformity of the hindfoot. However, most of coalitions in talocalcaneal middle facet are associated with rigid pes planovalgus. Simple resection is not suitable for all talocalcaneal coalition cases due to the variation in anatomy and deformity.

The traditional medial incision to the subtalar joint provides excellent exposure and resection of the coalition [34-37]. However, the traditional open technique had some disadvantages including the risk of incisional neuroma formation, superficial wound infection, delayed wound healing, and prolonged hospitalization for wound management and pain control [38].

Recently, posterior arthroscopic technique for posteriorfacet talocalcaneal coalition excision has been developed $[38,39]$. In the prone position, Bonasia introduced two portals, posterolateral (PL) portal and posteromedial (PM) portal for resection of talocalcaneal coalition [38]. Lee introduced a third portal, which was defined $1 \mathrm{~cm}$ proximal and $1 \mathrm{~cm}$ posterior to the tip of the lateral malleolus [40]. Beimers introduced another accessory portal, sinus tarsi portal, for introduction of a large diameter blunt trocar [41]. In the lateral position, generally anterior, medial, and posterior portal are used [42,43]. The anterior and middle subtalar portals were both less likely to damage the sural nerve and its branches than the posterior subtalar portal [44]. The arthroscopic procedure provides a better visualization of pathologic changes in the talocalcaneal joint and a complete resection of coalition $[45,46]$.

Senior authors (MAH and KLT) use a medial approach for an isolated coalition resection; and perform a dual lateral and medial approach for resection of the coalition and sinus tarsi arthrodesis, respectively (Figure 3A, 3B). We found no skin or nerve complications with either our isolated or dual incisional approaches. In conclusion, both open surgery and arthroscopy provide good exposure that facilitates predictable results.

The majority of patients with tarsal coalition showed a symptomatic relief and functional improvement in the short term after single resection of coalition [20,30,47]. However, patients with talocalcaneal coalition and flatfoot deformity may present with recurrent pain and worsening planovalgus deformities after a single resection of coalition. Hindfoot valgus and pes planovalgus deformities did not correct well after single resection of talocalcaneal coalitions $[33,48]$. These are due to the secondary effect of soft tissue contractures (lateral ligaments, peroneal tendons, calf muscles) pulling the foot into more valgus [49]. Normal plantar pressures were not recreated during running after resection of tarsal coalition [20,50]. In view of normal subtalar kinematics playing an important role with long-term implications; resection of the coalition combined deformity correction
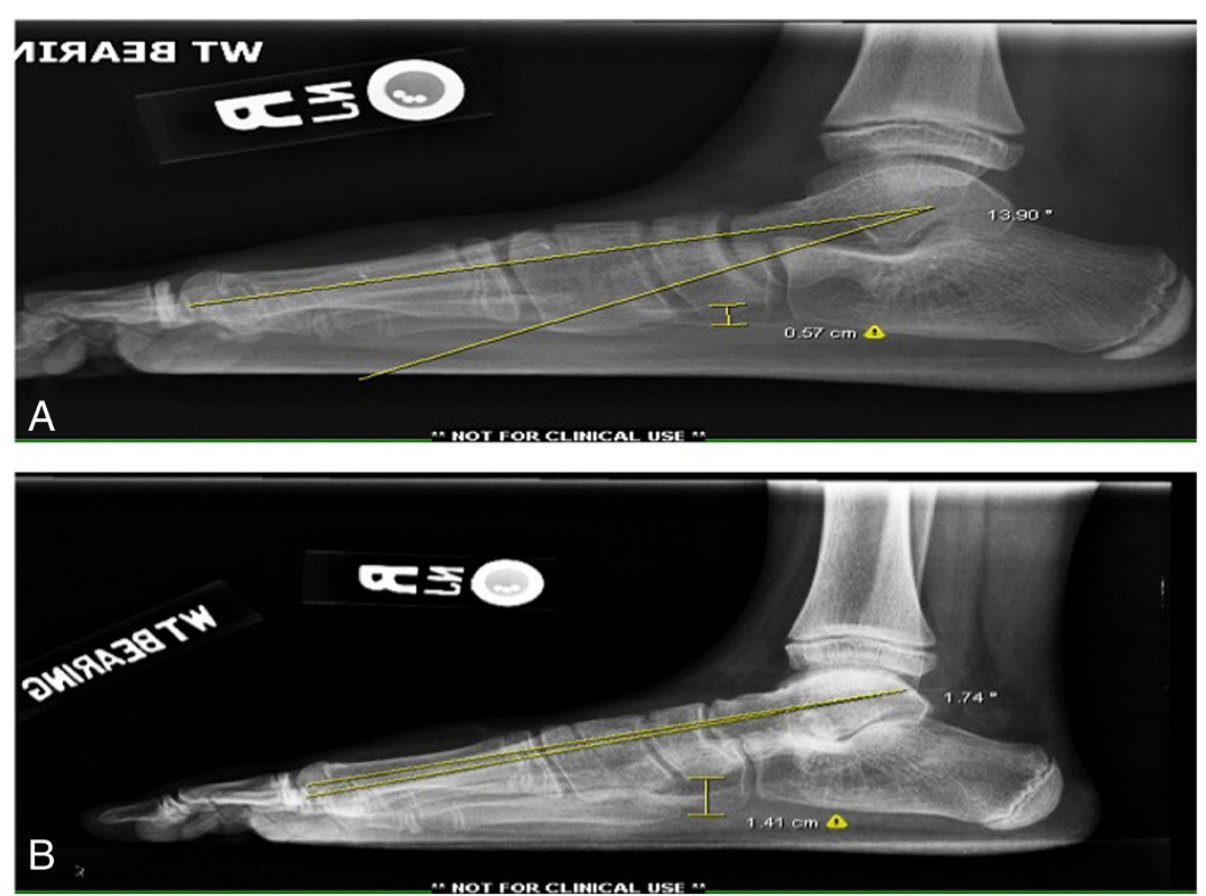

Figure 4 The weight-bearing lateral X-ray shows that the angle of the first tarsometatarsal and the height of the arch in the foot improved (A and $B)$. 
may be more appropriate in the patients, who had a talocalcaneal coalition with flatfoot deformity. In 1987, Olney performed excision of the coalition and an autogenous free fat graft for nine patients with a symptomatic talocalcaneal joint. Evaluation revealed five excellent, three good, one fair, and one poor result after 42 months follow-up postoperatively [51]. Interposition of the split flexor hallucis longus tendon resulted in considerable relief of pain, an improved range of motion of the talocalcaneal joint, and improved function of the foot after resection of talocalcaneal coalition $[52,53]$. In addition, an interposition with a tensor fascia lata allograft [54] or extensor digitorum brevis [55] or fibrin glue [56] achieved excellent or good results as well.

Single-stage middle facet talocalcaneal coalition resection combined with flatfoot reconstruction achieved a significant improvement in radiography and function of the foot [48]. Giannini reported that arthroereisis by implanting a bioreabsorbable device after resection of the tarsal coalition seems to restore the alignment of the hindfoot and reduce pain effectively for symptomatic flatfoot associated with talocalcaneal coalitions [57]. Arthroereisis functions by a combination of mechanical and proprioceptive effects that allow for growth adjustment of the subtalar joint and with a low complication rate. Though arthroereisis is contraindicated for treating fixed and secondary pes planovalgus [58]; the foot may gain mobility, albeit still limited, once resection of the talocalcaneal coalition has been performed. Gougoulias thought that gastrocnemius, Achilles, and/or peroneal tendon releases should be performed when the foot is in a planovalgus appearance, and also corrective calcaneal osteotomy may be required for young and adolescent patients with a flexible deformity to avoid equinus or further recurrence [49]. Mosca has reported that calcaneal lengthening osteotomy with gastrocnemius or Achilles tendon lengthening effectively corrected deformity and relieved pain in talocalcaneal coalition with planovalgus foot [59].

According to our experience, middle facet coalitions comprising more than $50 \%$ of the middle facet are best served with a subtalar joint arthrodesis. However, caution should be taken with primary arthrodesis of the entire talocalcaneal complex in children under the age of 13 due to possible growth disturbances of the hindfoot complex. An extra-articular arthrodesis is favored to prevent stunting of the normal hindfoot development. The Grice procedure has been originally designed by Grice in the treatment of children with polio [60]. Grice procedure performed extra-articular subtalar arthrodesis, which does not affect further bone growth and was widely used to correct spastic valgus deformity in children decades ago [61]. One of the senior authors (MAH) frequently performs modified extra-articular arthrodesis in osseous juvenile tarsal coalitions with the use of a Cloward spinal graft with much success (Figures 4 and 5).
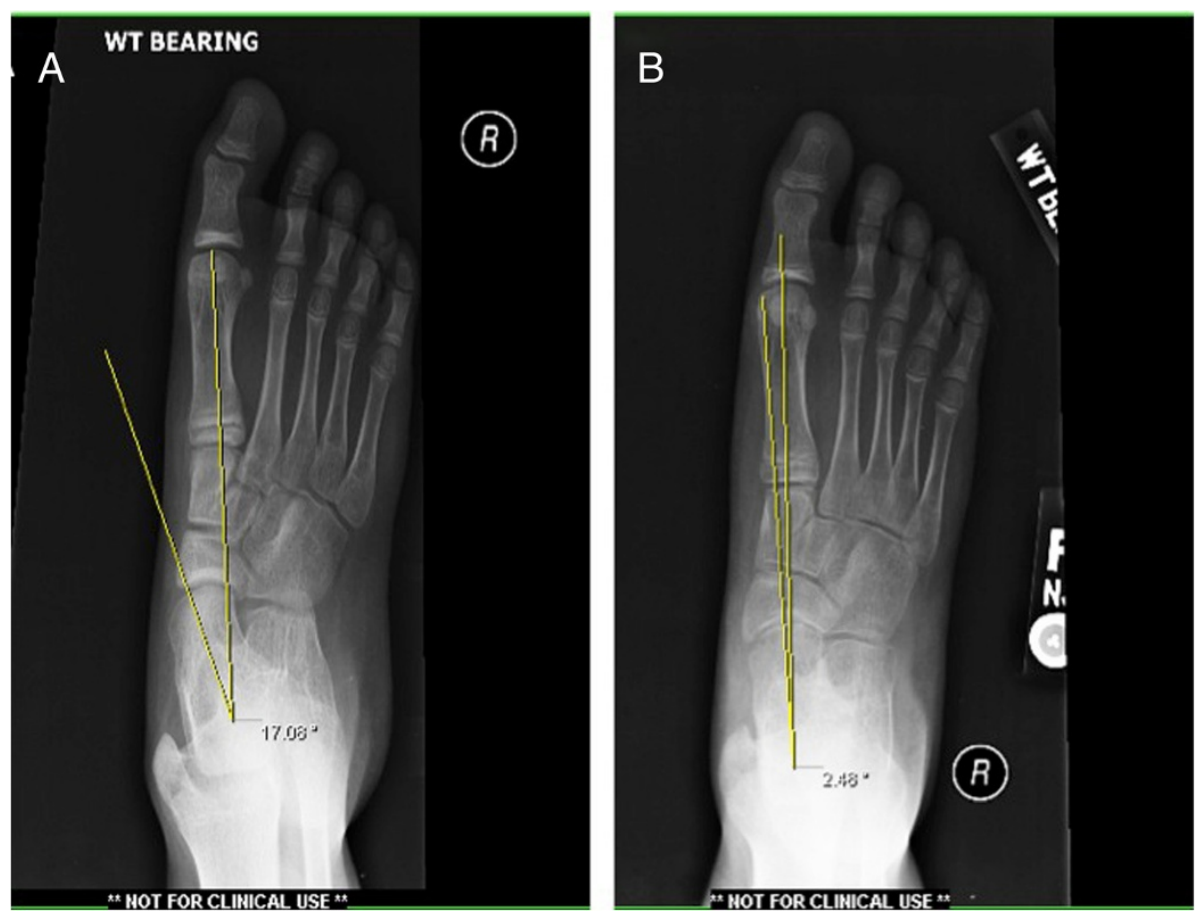

Figure 5 The weight-bearing anteroposterior X-ray shows that the angle of the first tarsometatarsal improves (A and B). 
In patients where less than $50 \%$ of the middle facet is involved, an attempt at salvage of the subtalar joint is preferred. This most often involves the resection of the coalition with placement of a subtalar joint arthroereisis to realign the hindfoot and preserve motion. Bone-block distraction was used in sinus tarsi arthrodesis. With bone-block distraction under the body of the talus, nearanatomical talar inclination is restored.

Collectively, nonoperative treatment may prove successful in children with talocalcaneal coalition with flatfoot. Resection of the coalition combined with an interpositional graft or arthroereisis or sinus arthrodesis has shown favorable outcomes. There is currently no clear consensus on the operative algorithm for talocalcaneal coalition with flatfoot. However, two operative criteria should be noticed for a long-term outcome: first, do not disturb the growth of the talocalcaneal joint; second, to correct the deformity combining resection of the coalition in a single-stage operation.

\section{Conclusion}

Talocalcaneal coalition with flatfoot is commonly seen in children. Talocalcaneal coalitions become increasing symptomatic as they ossify and restrict subtalar motion. Talocalcaneal coalition combined with a rigid flatfoot is characterized by some unique symptoms upon physical examination. Routine plain-film radiographs may detect talocalcaneal coalition combined with flatfoot effectively; however, advanced imaging such as CT and MRI may prove beneficial to delineate the etiology and guide surgical treatment. Conservative treatment options are useful and should be exhausted prior to entertaining surgical reconstruction. The optimal surgical procedure for talocalcaneal coalition with rigid flatfoot has not been definitively determined. However, combining resection of the coalition with concomitant flatfoot correction in a single-stage operation has shown to be valuable according to the long-term results.

\section{Competing interests}

The authors declare that they have no competing interests.

\section{Authors contributions}

$\mathrm{BHZ}$ collected the data and drafted the manuscript; MD and KLT conceived the idea and revised the manuscript. All authors read and approved the final manuscript.

\section{Acknowledgements}

We thank Dr. Jennifer Prezioso for the assistance in the polish of this manuscript.

Received: 16 August 2014 Accepted: 24 November 2014 Published online: 14 December 2014

\section{References}

1. Bohne WH: Tarsal coalition. Curr Opin Pediatr 2001, 13:29 35.

2. Lawrence DA, Rolen MF, Moukaddam H: Middle subtalar osseous coalition with associated fusion of the sinus tarsi: a previously undescribed type of tarsal coalition. Clin Imaging 2014, 38:67 69.
3. Snyder RB, Lipscomb AB, Johnston RK: The relationship of tarsal coalitions to ankle sprains in athletes. Am J Sports Med 1981, 9:313 317.

4. Stormont DM, Peterson HA: The relative incidence of tarsal coalition. Clin Orthop Relat Res 1983, 181:28 36.

5. Case $D$, Burnett $\mathrm{S}$ : Identification of tarsal coalition and frequency estimates from skeletal samples. Int J Osteoarchaeology 2012, 22:667 684

6. Lyon R, Liu X-C, Cho S-J: Effects of tarsal coalition resection on dynamic plantar pressures and electromyography of lower extremity muscles. J Foot Ankle Surg 2005, 44:252 258.

7. Cowell HR, Elener V: Rigid painful flatfoot secondary to tarsal coalition. Clin Orthop Relat Res 1983, 177:54 60.

8. Cass AD, Camasta CA: A review of tarsal coalition and pes planovalgus: clinical examination, diagnostic imaging, and surgical planning. J Foot Ankle Surg 2010, 49:274 293.

9. Warren MJ, Jeffree MA, Wilson DJ, Maclarnon JC: Computed tomography in suspected tarsal coalition: examination of 26 cases. Acta Orthop 1990, 61:554 557.

10. Jayakumar S, COWELL HR: Rigid flatfoot. Clin Orthop Relat Res 1977, 122:77 84 .

11. Kelo MJ, Riddle DL: Examination and management of a patient with tarsal coalition. Phys Ther 1998, 78:518 525.

12. Knapp HP, Tavakoli M, Levitz S, Sobel E: Tarsal coalition in an adult with cavovarus feet. J Am Podiatr Med Assoc 1998, 88:295 300.

13. Katayama T, Tanaka Y, Kadono K, Taniguchi A, Takakura Y: Talocalcaneal coalition: a case showing the ossification process. Foot Ankle Int 2005, 26:490 493

14. Leonard $\mathrm{M}$ : The inheritance of tarsal coalition and its relationship to spastic flat foot. J Bone Joint Surg Br 1974, 56:520 526.

15. Burnett S, Wilczak C: Tarsal and tarsometatarsal coalitions from Mound $C$ (Ocmulgee Macon Plateau site, Georgia): Implications for understanding the patterns, origins, and antiquity of pedal coalitions in Native American populations. Homo 2012, 63:167 181

16. Graham JM, Braddock SR, Mortier GR, Lachman R, Van Dop C, Jabs EW: Syndrome of coronal craniosynostosis with brachydactyly and carpal/ tarsal coalition due to Pro250Arg mutation in FGFR3 gene. Am J Med Genet 1998, 77:322 329.

17. Hetsroni I, Nyska M, Mann G, Rozenfeld G, Ayalon M: Subtalar kinematics following resection of tarsal coalition. Foot Ankle Int 2008, 29:1088 1094

18. Sakellariou A, Claridge RJ: Tarsal coalition: aetiology, diagnosis and treatment. Curr Orthop 1998, 12:135 142.

19. Kernbach KJ, Blitz NM: The presence of calcaneal fibular remodeling associated with middle facet talocalcaneal coalition: a retrospective CT review of 35 feet. Investigations involving middle facet coalitions part II. J Foot Ankle Surg 2008, 47:288 294.

20. Hetsroni I, Ayalon M, Mann G, Meyer G, Nyska M: Walking and running plantar pressure analysis before and after resection of tarsal coalition. Foot Ankle Int 2007, 28:575 580

21. Staser J, Karmazyn B, Lubicky J: Radiographic diagnosis of posterior facet talocalcaneal coalition. Pediatr Radiol 2007, 37:79 81.

22. Cooperman DR, Janke BE, Gilmore A, Latimer BM, Brinker MR, Thompson GH: A three-dimensional study of calcaneonavicular tarsal coalitions. J Pediatr Orthop 2001, 21:648 651 .

23. Bourdet C, Seringe R, Adamsbaum C, Glorion C, Wicart P: Flatfoot in children and adolescents. Analysis of imaging findings and therapeutic implications. Orthop Traumatol Surg Res 2013, 99:80 87.

24. Downey M: Tarsal coalitions. A surgical classification. J Am Podiatr Med Assoc 1991, 81:187 197.

25. Leerar PJ: Differential diagnosis of tarsal coalition versus cuboid syndrome in an adolescent athlete. J Orthop Sports Phys Ther 2001, 31:702 707.

26. Brown RR, Rosenberg ZS, Thornhill BA: The C sign: more specific for flatfoot deformity than subtalar coalition. Skeletal Radiol 2001, 30:84 87.

27. Crim JR, Kjeldsberg KM: Radiographic diagnosis of tarsal coalition. Am J Roentgenol 2004, 182:323 328.

28. Newman JS, Newberg AH: Congenital tarsal coalition: multimodality evaluation with emphasis on CT and MR imaging 1: (CME available in print version and on RSNA Link). Radiographics 2000, 20:321 332

29. Wilde P, Torode I, Dickens D, Cole W: Resection for symptomatic talocalcaneal coalition. J Bone Joint Surgery, British Volume 1994, 76:797 801

30. Thorpe SW, Wukich DK: Tarsal coalitions in the adult population: does treatment differ from the adolescent? Foot Ankle Clin 2012, 17:195 204 
31. Varner KE, Michelson JD: Tarsal coalition in adults. Foot Ankle Int 2000 21:669 672.

32. Hansen ST: Functional Reconstruction of the Foot and Ankle: Lippincott Williams \& Wilkins. 2000

33. Khoshbin A, Law PW, Caspi L, Wright JG: Long-term functional outcomes of resected tarsal coalitions. Foot Ankle Int 2013. doi:1071100713489122.

34. Anand P, Nunley JA, DeOrio JK: Single-incision medial approach for double arthrodesis of hindfoot in posterior tibialis tendon dysfunction. Foot Ankle Int 2013, 34:338 344.

35. Ahmad J, Pedowitz D: Management of the rigid arthritic flatfoot in adults: triple arthrodesis. Foot Ankle Clin 2012, 17:309 322.

36. Saville $P$, Longman CF, Srinivasan SC, Kothari P: Medial approach for hindfoot arthrodesis with a valgus deformity. Foot Ankle Int 2011, 32:818 821.

37. Jeng $C L$, Tankson CJ, Myerson MS: The single medial approach to triple arthrodesis: a cadaver study. Foot Ankle Int 2006, 27:1022 1025.

38. Bonasia DE, Phisitkul P, Saltzman CL, Barg A, Amendola A: Arthroscopic resection of talocalcaneal coalitions. Arthroscopy 2011, 27:430 435

39. Bernardino CM, Golano P, Garcia MA, Lopez-Vidriero E: Experimental model in cadavera of arthroscopic resection of calcaneonavicular coalition and its first in-vivo application: preliminary communication. J Pediatr Orthop 2009, 18:347 353 .

40. Lee KB, Saltzman CL, Suh JS, Wasserman L, Amendola A: A posterior 3-portal arthroscopic approach for isolated subtalar arthrodesis. Arthroscopy 2008, 24:1306 1310.

41. Beimers L, De Leeuw PA, Van Dijk CN: A 3-portal approach for arthroscopic subtalar arthrodesis. Knee Surg Sports Traumatol Arthrosc 2009, 17:830 834

42. Lui TH: Arthroscopic subtalar release of post-traumatic subtalar stiffness. Arthroscopy 2006, 22:1364. e1361-1364.

43. Frey C, Gasser S, Feder K: Arthroscopy of the subtalar joint. Foot Ankle Int 1994, 15:424 428.

44. Tryfonidis M, Whitfield CG, Charalambous CP, Baraza WK, Blundell C, Sharp RJ: The distance between the sural nerve and ideal portal placements in lateral subtalar arthroscopy: a cadaveric study. Foot Ankle Int 2008, 29:842 844.

45. Lintz F, Guillard C, Colin F, Marchand J-B, Brilhault J: Safety and efficiency of a 2-portal lateral approach to arthroscopic subtalar arthrodesis: a cadaveric study. Arthroscopy 2013, 29:1217 1223.

46. Bauer T, Golano P, Hardy P: Endoscopic resection of a calcaneonavicular coalition. Knee Surg Sports Traumatol Arthrosc 2010, 18:669 672.

47. Philbin TM, Homan B, Hill K, Berlet G: Results of resection for middle facet tarsal coalitions in adults. Foot Ankle Spec 2008, 1:344 349.

48. Kernbach KJ, Blitz NM, Rush SM: Bilateral single-stage middle facet talocalcaneal coalition resection combined with flatfoot reconstruction: a report of 3 cases and review of the literature. Investigations involving middle facet coalitions part 1. J Foot Ankle Surg 2008, 47:180 190.

49. Gougoulias N, O Flaherty M, Sakellariou A: Taking out the tarsal coalition was easy: but now the foot is even flatter. What now? Foot Ankle Clin 2014, 19:555 568.

50. Skwara A, Zounta V, Tibesku CO, Fuchs-Winkelmann S, Rosenbaum D: Plantar contact stress and gait analysis after resection of tarsal coalition. Acta Orthop Belg 2009, 75:654.

51. Olney BW, Asher M: Excision of symptomatic coalition of the middle facet of the talocalcaneal joint. J Bone Joint Surgery 1987, 69:539 544.

52. Raikin S, Cooperman DR, Thompson GH: Interposition of the split flexor hallucis longus tendon after resection of a coalition of the middle facet of the talocalcaneal Joint*. J Bone Joint Surgery 1999, $81: 1119$

53. Kumar S, Guille J, Lee M, Couto J: Osseous and non-osseous coalition of the middle facet of the talocalcaneal joint. J Bone Joint Surg Am 1992, 74:529 535

54. Di Liddo PE, Rivard DS, Mehler AS, Wertheimer SJ: Resection of talocalcaneal middle facet coalition. Interposition with a tensor fascia lata allograft: a case report. J Foot Ankle Surg 2000, 39:336 340.

55. Digitorum E: Calcaneonavicular coalition treated by resection and interposition of the extensor digitorum brevis muscle. J Bone Joint Surg Am 1990, 72:71 77.

56. Weatherall JM, Price AE: Fibrin glue as interposition graft for tarsal coalition. Am J Orthop 2013, 42:26 29.

57. Giannini S, Ceccarelli F, Vannini F, Baldi E: Operative treatment of flatfoot with talocalcaneal coalition. Clin Orthop Relat Res 2003, 411:178 187.
58. Abbara-Czardybon M, Wingenfeld $C$, Arbab D, Frank D: Options and limits of subtalar arthroereisis in childhood. Orthopade 2013, 42:12 19

59. Mosca VS, Bevan WP: Talocalcaneal tarsal coalitions and the calcaneal lengthening osteotomy: the role of deformity correction. J Bone Joint Surgery 2012, 94:1584 1594

60. Grice DS: Ax extra-articular arthrodesis of the subastragalar. Joint 1952 34:927 956

61. Hiness PR, Kirkhus E: Grice arthrodesis in the treatment of valgus feet in children with myelomeningocele: a 12.8-year follow-up study. J child Orthop 2009, 3:283 290.

doi:10.1186/s13018-014-0129-9

Cite this article as: Zhou et al:: Talocalcaneal coalition combined with

flatfoot in children: diagnosis and treatment: a review. Journal of Orthopaedic Surgery and Research 2014 9:129.

\section{Submit your next manuscript to BioMed Central and take full advantage of:}

$\otimes$ Convenient online submission

$\otimes$ Thorough peer review

$\otimes$ No space constraints or color $\nabla$ gure charges

$\nabla$ Immediate publication on acceptance

Q Inclusion in PubMed, CAS, Scopus and Google Scholar

$\nabla$ Research which is freely available for redistribution

Submit your manuscript at www.biomedcentral.com/submit
C Biomed Central 